\title{
Cloud Monitoring for Solar Plants with Support Vector Machine Based Fault Detection System
}

\author{
Hong-Chan Chang, ${ }^{1}$ Shang-Chih Lin, ${ }^{1}$ Cheng-Chien Kuo, ${ }^{2}$ and Hao-Ping Yu ${ }^{1}$ \\ ${ }^{1}$ Department of Electrical Engineering, National Taiwan University of Science and Technology, No. 43, Section 4, \\ Keelung Road, Taipei 10607, Taiwan \\ ${ }^{2}$ Department of Electrical Engineering, Saint John's University, No. 499, Section 4, TamKing Road, Tamsui, Taipei 25135, Taiwan
}

Correspondence should be addressed to Cheng-Chien Kuo; cckuo@mail.sju.edu.tw

Received 28 February 2014; Accepted 4 April 2014; Published 3 July 2014

Academic Editor: Her-Terng Yau

Copyright (C) 2014 Hong-Chan Chang et al. This is an open access article distributed under the Creative Commons Attribution License, which permits unrestricted use, distribution, and reproduction in any medium, provided the original work is properly cited.

\begin{abstract}
This study endeavors to develop a cloud monitoring system for solar plants. This system incorporates numerous subsystems, such as a geographic information system, an instantaneous power-consumption information system, a reporting system, and a failure diagnosis system. Visual C\# was integrated with ASP.NET and SQL technologies for the proposed monitoring system. A user interface for database management system was developed to enable users to access solar power information and management systems. In addition, by using peer-to-peer (P2P) streaming technology and audio/video encoding/decoding technology, real-time video data can be transmitted to the client end, providing instantaneous and direct information. Regarding smart failure diagnosis, the proposed system employs the support vector machine (SVM) theory to train failure mathematical models. The solar power data are provided to the SVM for analysis in order to determine the failure types and subsequently eliminate failures at an early stage. The cloud energy-management platform developed in this study not only enhances the management and maintenance efficiency of solar power plants but also increases the market competitiveness of solar power generation and renewable energy.
\end{abstract}

\section{Introduction}

Green energy is universally regarded as an alternative energy for the future. The production and consumption of green energy slightly pollute or do not pollute ecological environments, and it will not be exhausted. Therefore, various advanced countries pay increasing attention to green energy, and Taiwan continually pushes green energy plans in accordance with the global trend. Therefore, the green energy of solar power generation and wind electric power generation will be extensively used in the future. This paper is aimed at this future trend and proposes a solar energy monitoring and fault diagnosis system based on the cloud structure, which is combined with power consumption information to provide a professional and complete electrical energy management service.

This electric energy management platform architecture approximately consists of three basic structures, including data collection, data storage, and data application. First, for the data collection layer, this electric energy management platform will be equipped with an environmental monitoring system, a power monitoring system for solar power generation, GPS, and communication system. The most important information for the environmental monitoring system includes temperature, humidity, and luminance. Therefore, the environmental monitoring system requires corresponding sensors to extract relevant information. For the power monitoring system of solar power generation, the sensors must be mounted on the solar inverter in order to extract the power generation data of solar panels. Each of the aforesaid subsystems consists of numerous sensors and communication equipment. The data measured by all sensors are transmitted by the communication system to the relay station, where the data received by the relay station are uploaded via network to the data storage center. The data storage center uses database technology to store the data collected by each subsystem. Professional software technology is required for data storage in order to maintain data acquisition speed 


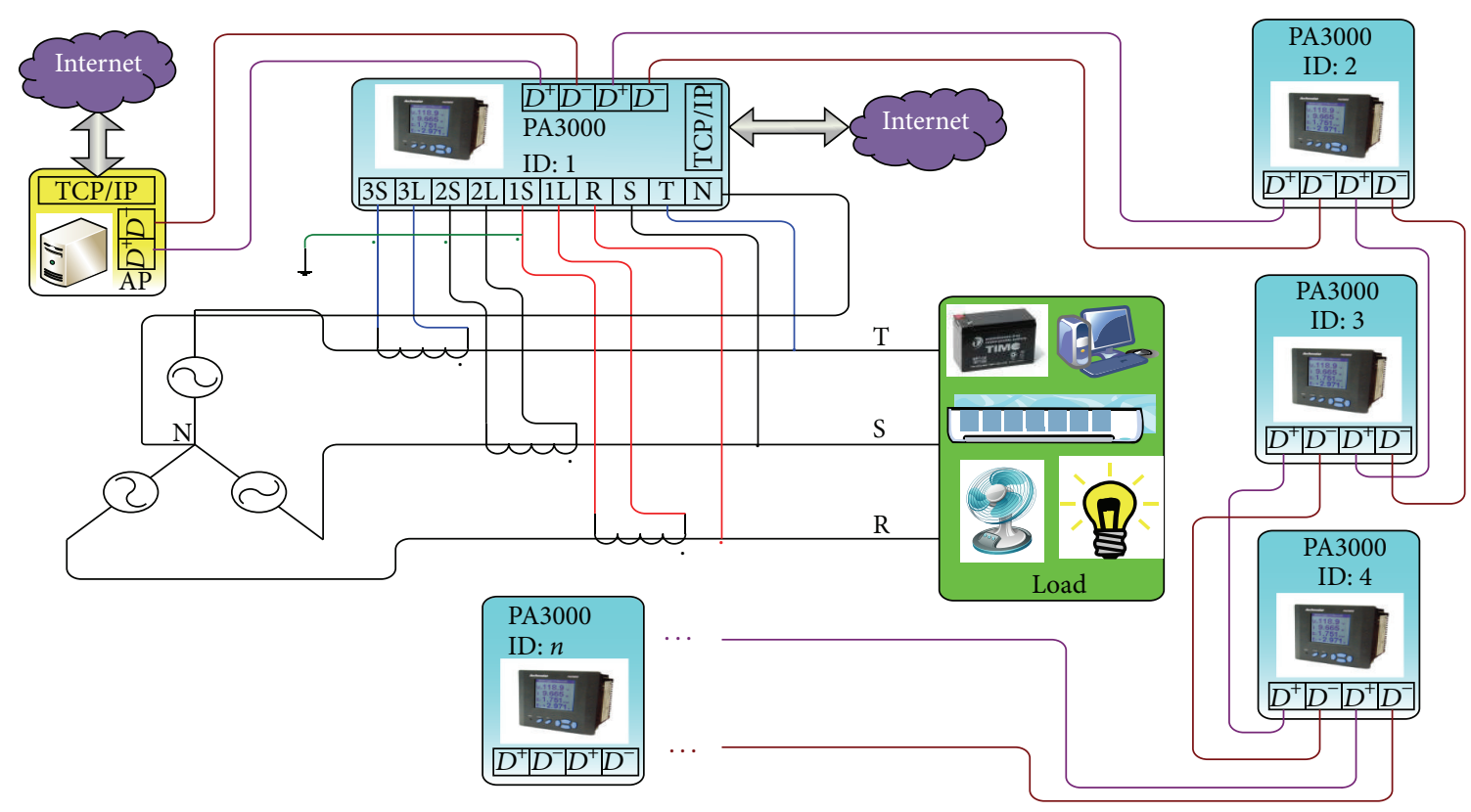

FIGURE 1: Hardware architecture.

and security. Finally, this electric energy management system uses software technology to convert the data detected by various equipment into useful information, thus providing electric energy management services of failure diagnosis and equipment operating state for solar power plants.

\section{Cloud Structure-Based Monitoring System}

2.1. Hardware Architecture. The hardware architecture for electric energy measurement in this system takes the threephase four-wire connection mode as the basis of measurement, as shown in Figure 1. A three-phase Y-connection power supply is connected to the load, and the R, S, and T phases incorporate a transformer, respectively, and connect the smart meter PA3000 of the Archmeter company to measure various electric quantities. Each set of solar inverters is equipped with PA3000 to detect the power status of each inverter. As a solar power plant consists of multiple distributed solar modules and each module is equipped with an inverter, the communication system must allow multiple devices to be connected in the same network for communication. Therefore, the PA3000, which supports the Modbus communication protocol, is adopted. The Modbus of PA3000 provides two communication protocol versions. One is the common RS-485 communication standard of industrial circles, where there are generally 32 measurement systems connected; however, the distance cannot exceed $1.2 \mathrm{~km}$. If the transmission distance exceeds $1.2 \mathrm{~km}$, there will be an additional RS-485 relay station to avoid omitting signal packets; the other one uses the internet to send data. If there is a network at the measurement site and each set of measuring devices has a corresponding fixed IP, the Ethernet of TCP/IP communication standard can be connected, which enables this system to remotely read electric energy data.
2.2. Data Acquisition System. The reliability of the monitoring system depends on the accuracy and integrity of data acquisition system. Therefore, how to select the measurement system and communication technology is a very important topic. The main operation process of the data acquisition system is described as follows. First, the signals from the remote sensor are converted and preprocessed into signals with the best resolution, which can be processed by the analog-todigital conversion circuit, and then the digitized raw data are transmitted to the microcomputer, which determines the values of physical quantities and sends the data via hardware line and communication protocol to the local side. Finally, the results are displayed on the human-machine interface as shown in Figure 2.

The program flow of the data acquisition system is as shown in Figure 3. When the program is executed, all COM Port connection states are read, and any new COM Port connection is detected. The COM Port is switched on, automatic reading is set, and the system automatically reads electric energy data every 15 minutes. In the process of reading, the packet missing detection is started, and packet data integrity is determined. If the packets have no issue, the data are uploaded to the local database and remote database and displayed on the data interface.

2.3. Data Acquisition Method. As a solar power plant consists of multiple distributed solar modules and each module is equipped with an inverter, the communication system must allow multiple devices to be connected in the same network for communication. The Modbus allows multiple devices to be connected in the same network for communication. Therefore, this data acquisition system uses the common Modbus communication protocol in industry. The Modbus communication protocol is a communication protocol developed by the Modicon Company and is usually applied to 


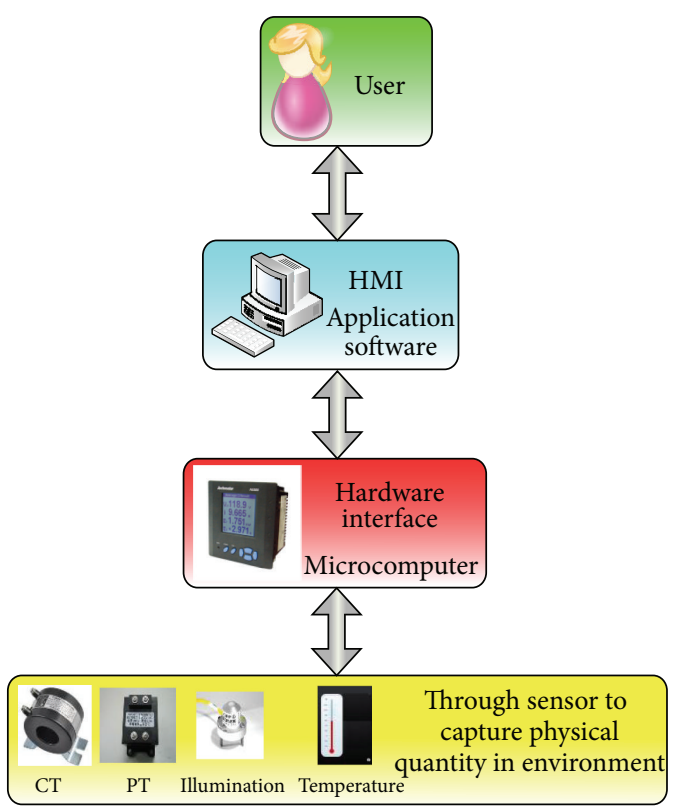

Figure 2: Data acquisition architecture.

the serial port and Ethernet communication architecture. Its advantage is that the communication protocol content is completely open, is free of authorization fees, and is easily operated, and the conformability of different equipment is high. Therefore, the development schedule of a larger system will be shortened, thus reducing the cost.

The Modbus communication protocol is master-slave architecture, as shown in Figure 4. The overall architecture has only one master, that is, the data acquisition system, while the remaining are slaves, that is, measurement systems, and each slave will have one unique address in order that the master can accurately receive the corresponding data of each slave during inquiry.

2.4. Cloud System. Figure 5 shows the cloud system software architecture [1-4], including (1) local data center, (2) login management server, (3) real-time video streaming server, (4) video streaming transmission function, and (5) cloud data center, described as shown in Figure 5.

(1) Local data center: the electric energy data collection program collects the data about the current operating state and power consumption of various subsystems via RS-485 or TCP/IP communication interface and Modbus communication protocol and uploads these parameters to the local data center and cloud data center. Afterwards, the client-side can use a browser to inquire and set relevant information, via the network, anywhere at any time, and can observe the present situation through the real-time video system.

(2) Login management server: the login management server divides authorities into administrator and user. The account and password are entered in the login page to contact the login management server. The user or administrator credentials are fed back after the identity is confirmed, thus giving the client-side
TABLE 1: Solar power system failure causes.

\begin{tabular}{ll}
\hline General failure type & Specific failure \\
\hline \multirow{2}{*}{ Long-term energy loss } & Solar cell aging \\
& Solar cell dirtiness \\
& Solar cell defect \\
& Solar cell connection defect \\
\hline \multirow{3}{*}{ Sudden change in energy loss } & Shadow \\
& High temperature \\
& Power grid off \\
\hline \multirow{2}{*}{ Power off } & Inverter defect \\
& Control device defect \\
\hline
\end{tabular}

different authorities, and the user and administrator have respective working environments.

(3) Real-time video streaming server: in terms of streaming transmission, this system uses the DirectShow development tool of the VLC multimedia player to extract live video from the video input device. The video data are encoded using the video encoding technique. Finally, streaming transmission is adopted for the encoded video data.

(4) Video streaming transmission function: the video streaming transmission function in this paper uses SQL database for communication between clientside and real-time video streaming server. When the client-side is given server-side authority and requires a real-time video source, the server-side confirms the client-side authority in SQL database and then removes the real-time video source which it sends to the client-side in order that the client-side can receive the real-time video streaming service of the real-time video system.

(5) Cloud data center: this cloud data center stores the data from different places, provided with ASP.NET server-side grammar C\# and client-side grammar HTML and JavaScript, and controls XML grammar and SQL database grammar between them. The communication among client-side, server-side, and database forms a cloud structure-based monitoring system, thus becoming a huge cloud data center.

\section{Solar Energy Fault Diagnosis System}

3.1. Introduction and Analysis of Failure Types. The solar power system failure causes are listed in Table 1, including long-term energy loss, sudden change in energy loss, and power off. Please refer to $[5,6]$ for specific descriptions of failures.

According to statistical data and published studies, the highest probability of the aforesaid failures is the high temperature failure caused by long-term covering. Therefore, this system proposes a failure diagnosis method using the mathematical model generated by training failure data to identify whether there are failure symptoms or not and intending to determine the problems in the solar power system early, thus prolonging the lifetime and efficiency of the solar power system. 


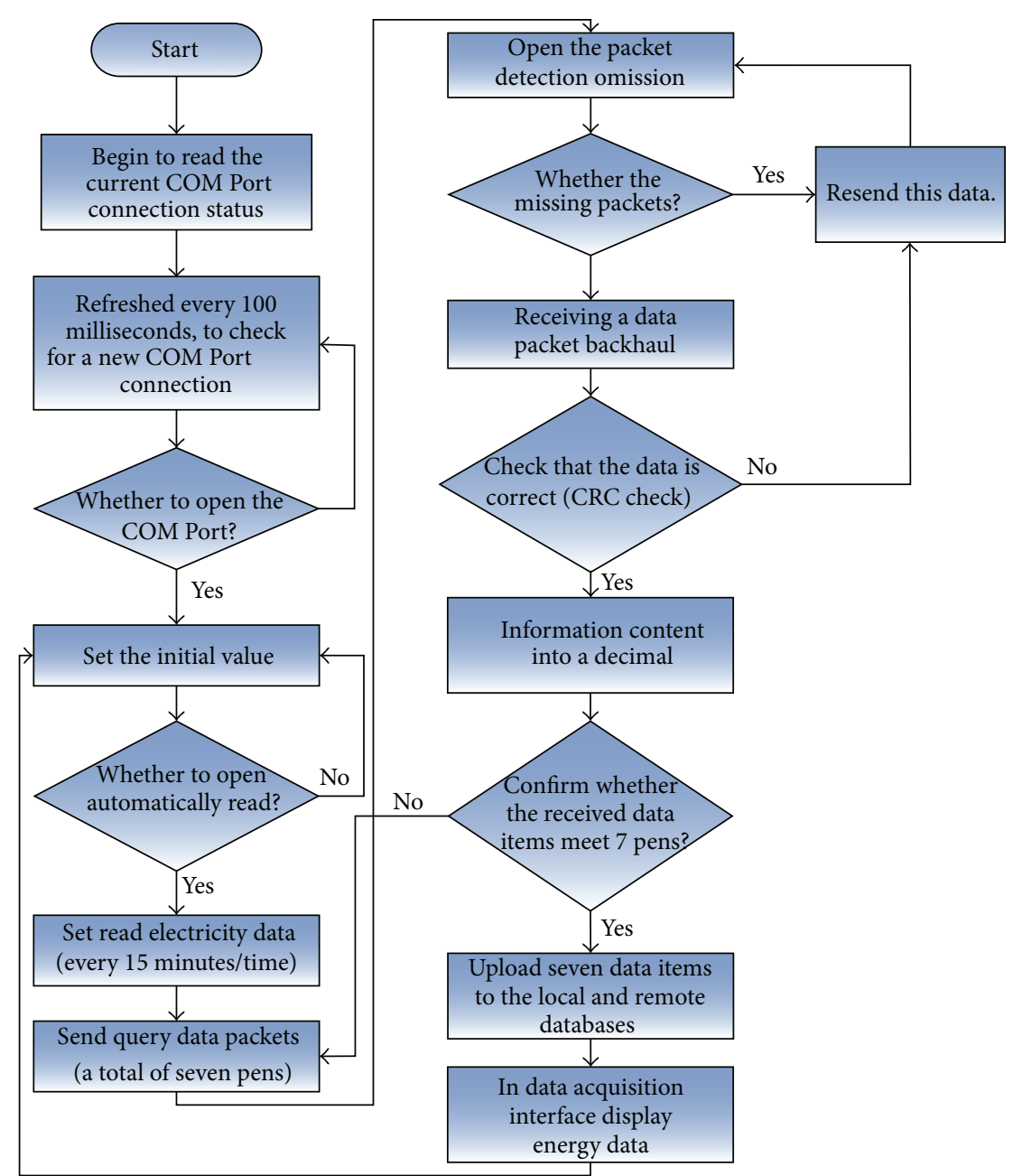

FIGURE 3: Data acquisition system program flow chart.

3.2. High Temperature Failure Analysis. The trees, buildings, black clouds, electric poles, and cables around the solar array result in different illumination angles shading the solar array, or possibly bird droppings or smudges on the solar cell module result in partial shading of the solar cell; such factors usually cause high temperature failure, with the principle as shown in Figures 6 and 7. Figure 6 shows the series circuit of $n$ solar cells. The passing current is the same if all the solar cells are not shaded, which is the normal power generation state; on the contrary, as shown in Figure 7, if No. $n$ solar cell is shaded, the $I_{p h}$ of the cell is zero; however, as the cells are connected in series, the total current must flow through No. $n R_{p}$ before it reaches the load. At this point, the diode voltage has reverse bias due to resistor $R_{p}$ voltage; thus, there is no through current. As the solar cell is regarded as load and consumes the electric energy generated by other solar cells, there is a great deal of thermal energy generated, and as the overall solar cell module is heated, the solar power generating efficiency decreases. In addition, the long-term cumulative temperature may influence the overall solar module structure, which damages the internal physical characteristics and packaging material [7].
3.3. Solar Cell Modeling. As it is difficult to obtain the practical failure data of a solar power system, in order to validate the feasibility of this failure diagnosis system, this paper builds a model to obtain the simulation data of various failures and normal conditions of the solar power system in order to develop and validate the failure diagnosis system. As the solar cell is a nonlinear DC power supply, its voltage and current characteristics are influenced by the solar illumination intensity and work environment temperature. In order to validate the feasibility of this failure diagnosis system, the solar cell module is built in a MatLab/Simulink environment, as shown in Figure 8. S is the input solar illumination intensity, $T$ is ambient temperature, and Out+ and Out- are the two output voltage ends of the solar cell.

This simulation refers to the module provided in the literature [8], which used a $50 \mathrm{~W}$ solar cell module as the simulated subject and used the parameters provided by the firm in Table 2 and S-function to build a solar cell model similar to an actual power generation module.

3.4. Principle of SVM. The SVM is developed from the optimal classification plane in the case of linear separability, as well as the most practical part of statistical learning theory 


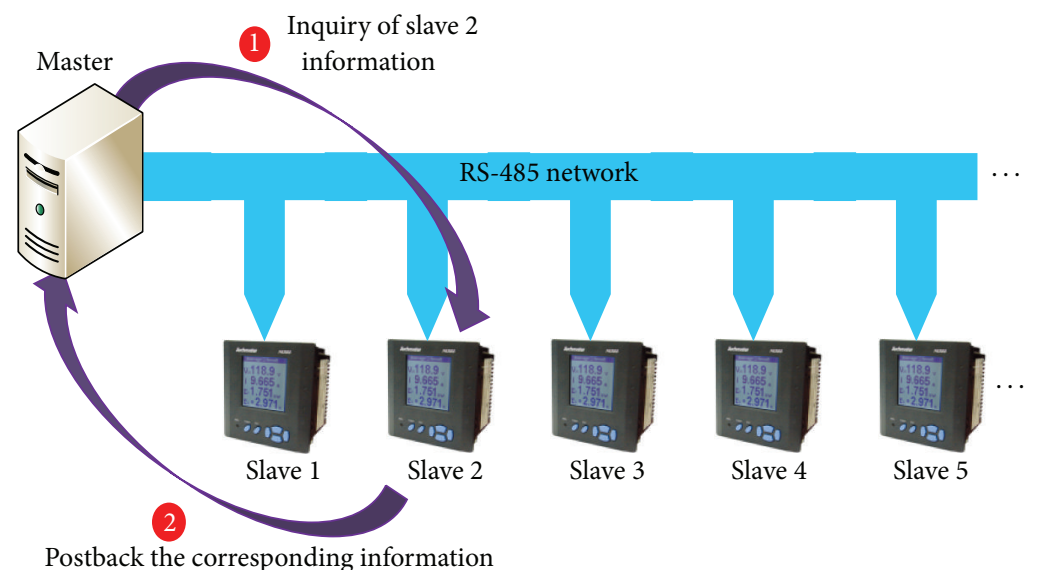

FIGURE 4: Master-slave architecture.

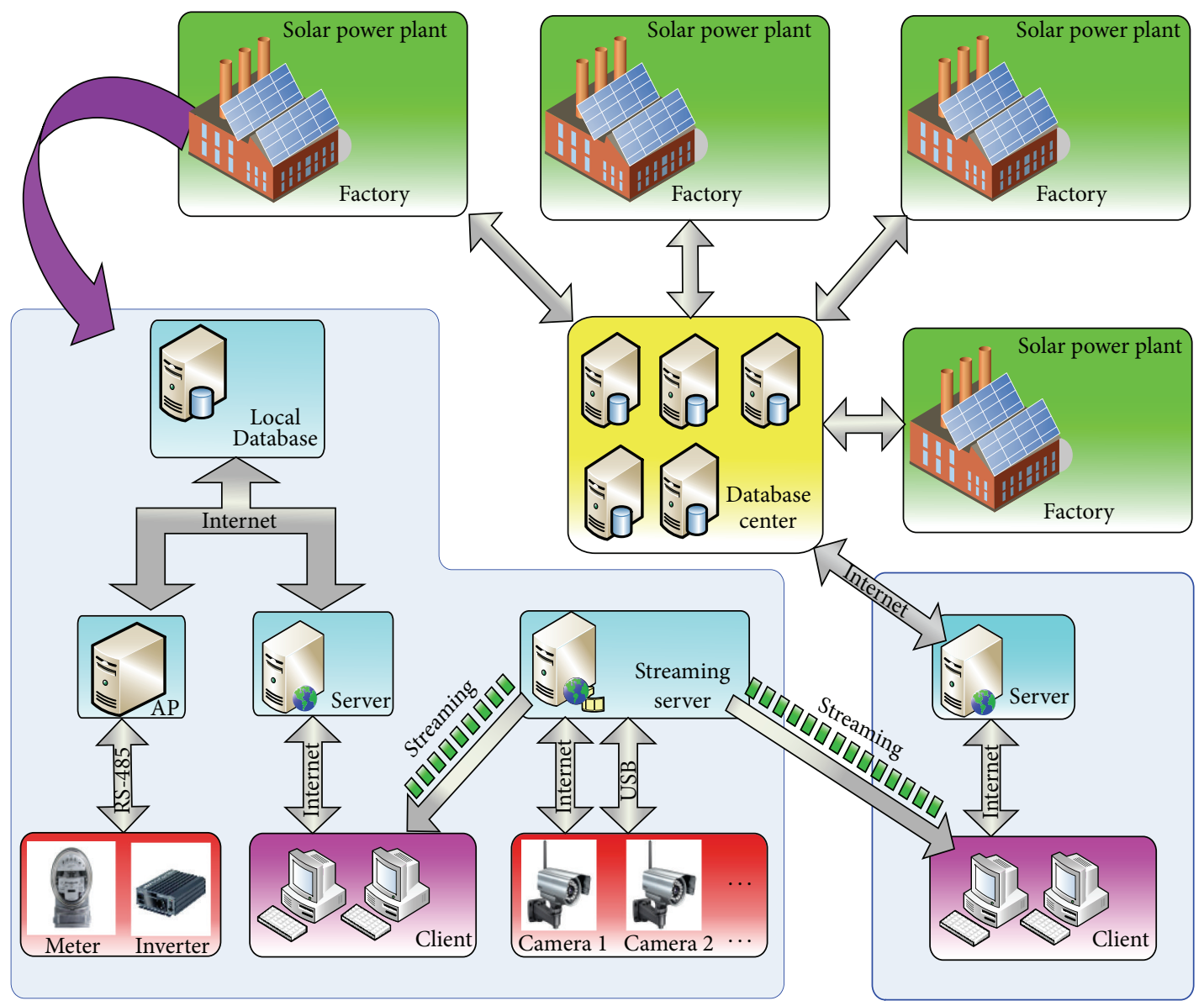

FIgURE 5: Cloud system software architecture.

[9-12]. Its basic principle can be described by the twodimensional condition in Figure 9. " $\mathrm{X}$ " and "O" represent negative and positive samples, $\mathrm{H}$ is the classification hyperplane, and $\mathrm{H} 1$ and $\mathrm{H} 2$ are the planes closest to classification hyperplane and parallel to classification hyperplane in respective classification, and their distance is called margin. The optimal classification plane means the classification plane is required not only to separate two classes correctly (i.e., training error rate is 0 ), but also to maximize the margin. The vector closest to the optimal classification hyperplane is called a support vector [13].

3.5. Shading Failure Training Method. The shading problem discussed in this paper assumes that one solar module of the solar array is shaded by external canvas, a plastic bag, or branches, as shown in Figure 10. The severity of shading is 


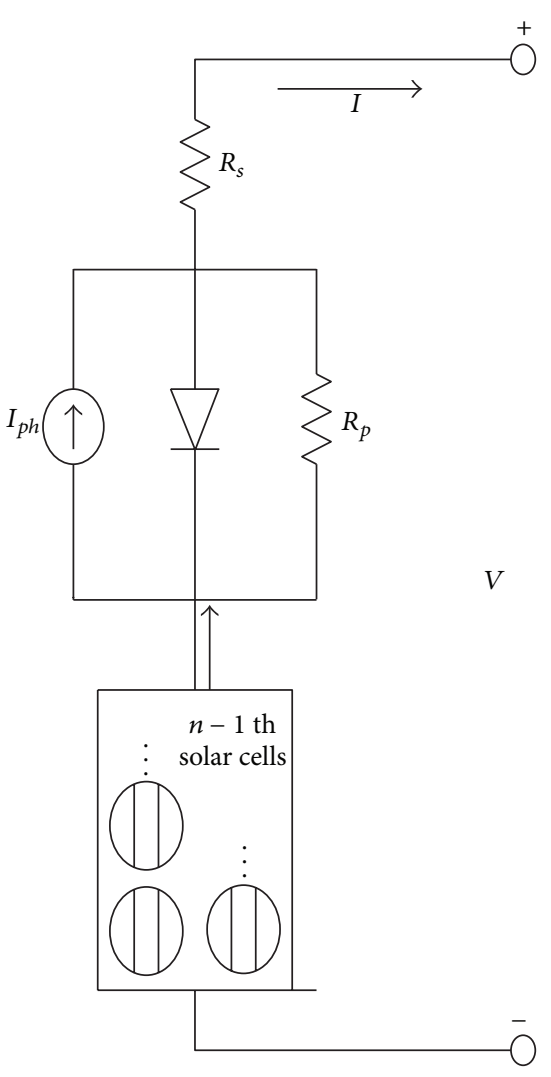

Figure 6: Unshaded solar cell.

TABLE 2: Electric energy parameters of solar module.

\begin{tabular}{lcc}
\hline Parameter & Symbol & Value \\
\hline Maximum power & $P_{\mathrm{MPP}}$ & $50 \mathrm{~W}$ \\
Maximum power voltage & $V_{\mathrm{MPP}}$ & $17.98 \mathrm{~V}$ \\
Maximum power current & $V_{\mathrm{MPP}}$ & $2.77 \mathrm{~A}$ \\
Short-circuit current & $I_{\mathrm{SC}}$ & $3 \mathrm{~A}$ \\
Open-circuit voltage & $V_{\mathrm{OC}}$ & $22 \mathrm{~V}$ \\
Short-circuit current temperature coefficient & $\alpha$ & $0.04 \% /{ }^{\circ} \mathrm{C}$ \\
Open-circuit current temperature coefficient & $\beta$ & $-0.33 \% /{ }^{\circ} \mathrm{C}$ \\
\hline
\end{tabular}

analyzed according to the transparence of the shade, and the feasibility of SVM to this system is validated.

This system defines the $0 \% \sim 25 \%$ shading range as a positive sample and the $25 \%$ 100\% shading range as a negative sample. The illuminance of various solar panels and the electric energy parameters (terminal voltage, power, and current of various solar panels), as generated by simulated solar array in different illuminance combination conditions, are collected as samples. In the simulated solar array, the sample data training SVM are obtained in the following ways.

(1) Positive sample: the illuminance of eight solar panels is set in the illuminance pattern of $1000 \mathrm{~W} / \mathrm{m}^{2}$ decreasing at a fixed slope, with the time as shown in Figure 11; the illuminance of the other panel is set as $100 \%, 90 \%, 80 \%$, and $75 \%$ of the illuminance of the aforesaid eight modules in turn,

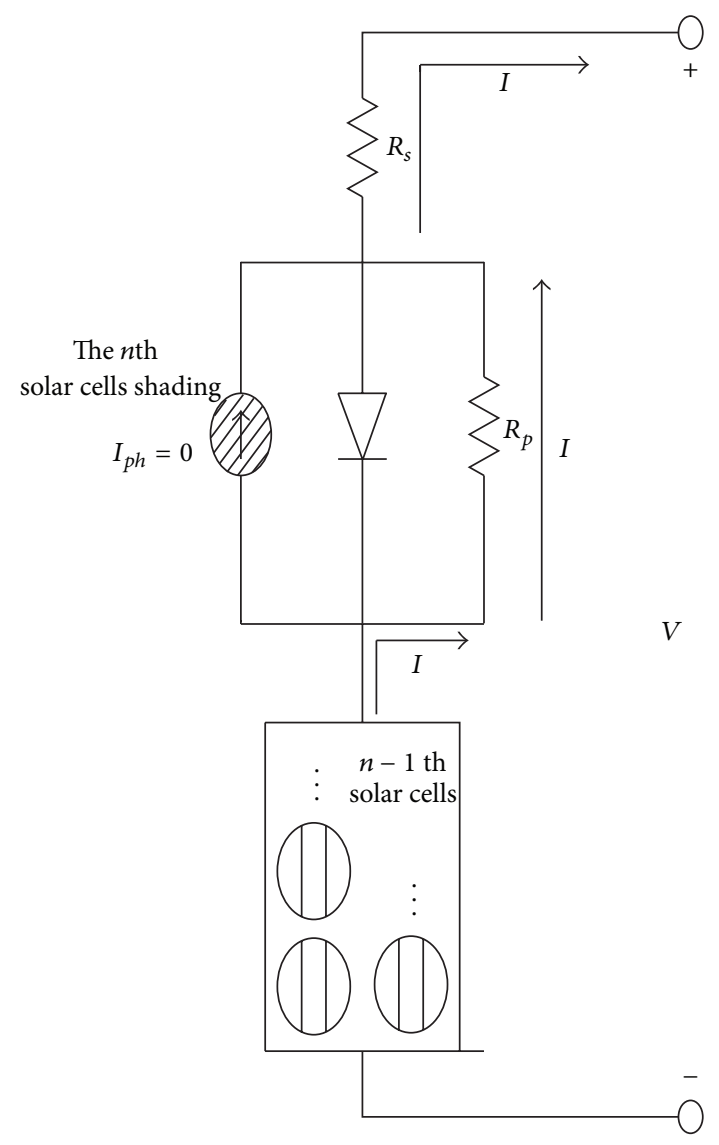

FIgURE 7: No. $n$ shaded.

and the sample data of various combinations are read.

(2) Various solar panels shaded negative sample: the illuminance of eight solar panels is set by the illuminance of $1000 \mathrm{~W} / \mathrm{m}^{2}$ decreasing at a fixed slope, with the time as shown in Figure 11; the illuminance of one panel of the first string of solar panels is set as $70 \%, 60 \%, 50 \%, 40 \%, 30 \%, 20 \%, 10 \%$, and $0 \%$ of the illuminance of the aforesaid eight modules in turn, and the sample data of various combinations are read.

Afterwards, the sample data obtained from different illuminance combinations are given to the SVM to execute shading failure model training.

\section{System Representation and Experimental Results}

4.1. Data Acquisition System Interface. An electric energy collection program with data acquisition function is developed at the central monitoring end of this system (Figure 12). The program provides a simple window software control interface; thus, users can learn how to operate this interface without spending too much time and implement monitoring and uploading data to the data center. The software interface contains data packet transfer, receiving display control 


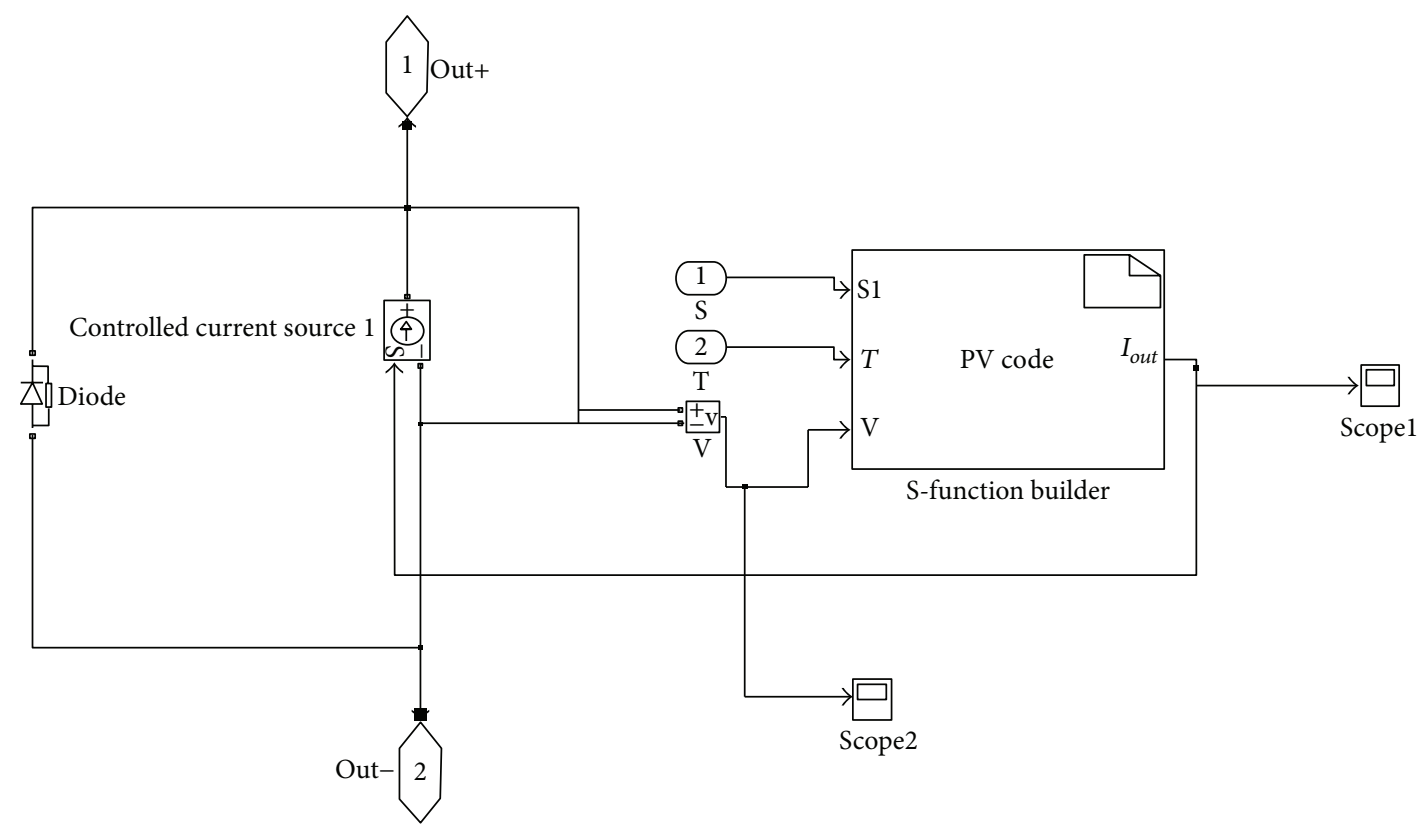

FIGURE 8: Solar cell module.

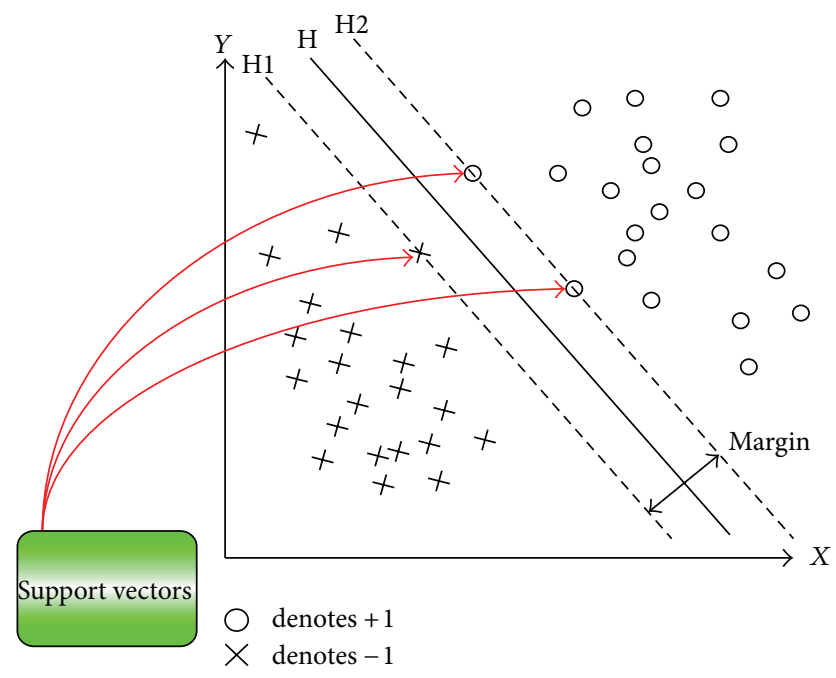

FIGURE 9: Classification hyperplane.

interface, and serial transmission setting interface, while other tag pages contain a three-phase voltage and current and electric energy information display interface, a total harmonic distortion display interface, and a three-phase voltage and current harmonic distortion display interface. This system takes laboratory power consumption conditions as the measured object at present.

\subsection{Cloud System}

4.2.1. Real-Time Power Consumption Monitoring System. Figure 13 shows the previous inquiry of power consumption, with graphically measured electric energy parameters listed and displayed in order that the client-side is aware of the present power consumption information. The list of this system is divided into two parts. One shows the current, voltage, active power, reactive power, apparent power, voltage angle, and current angle of the three phases. The other shows the average or total electric energy parameters of the three phases. The electric energy parameters measured in the last 24 hours, three days, or seven days can be inquired, which allows the user to identify data on the list or graphical interface, providing a more human-friendly query interface.

4.2.2. Real-Time Video System. In addition to remote data monitoring, in order to approach real-time monitoring and master local real-time conditions, this system is integrated with a real-time video function. To implement this real-time video function, a streaming server is required to collect all the real-time video data and to transfer these data by streaming output to the client-side connected computer, as shown in Figure 14. The program is a video capture and streaming program, which resides in the streaming server. The input video source is selected before the IP address of the streaming server and the Port number for video output are set, and then video encoding mode, transmission bit rate, and scaling are selected. Finally, the play button is clicked to run the video streaming function.

If the client-side is connected to this server, via a network, in order to watch the real-time video, the real-time video system is entered, as shown in Figure 15. When the video to be watched is clicked, the server inquires the IP address and Port number corresponding to the present streaming server in the database and then transfers the IP address and Port number to the client-side. When the client-side master receives the connection data, it looks for and connects the video source of streaming server according to the data, which it transfers to the client-side via the network. Finally, the client-side can watch the video content instantly for real-time video. 


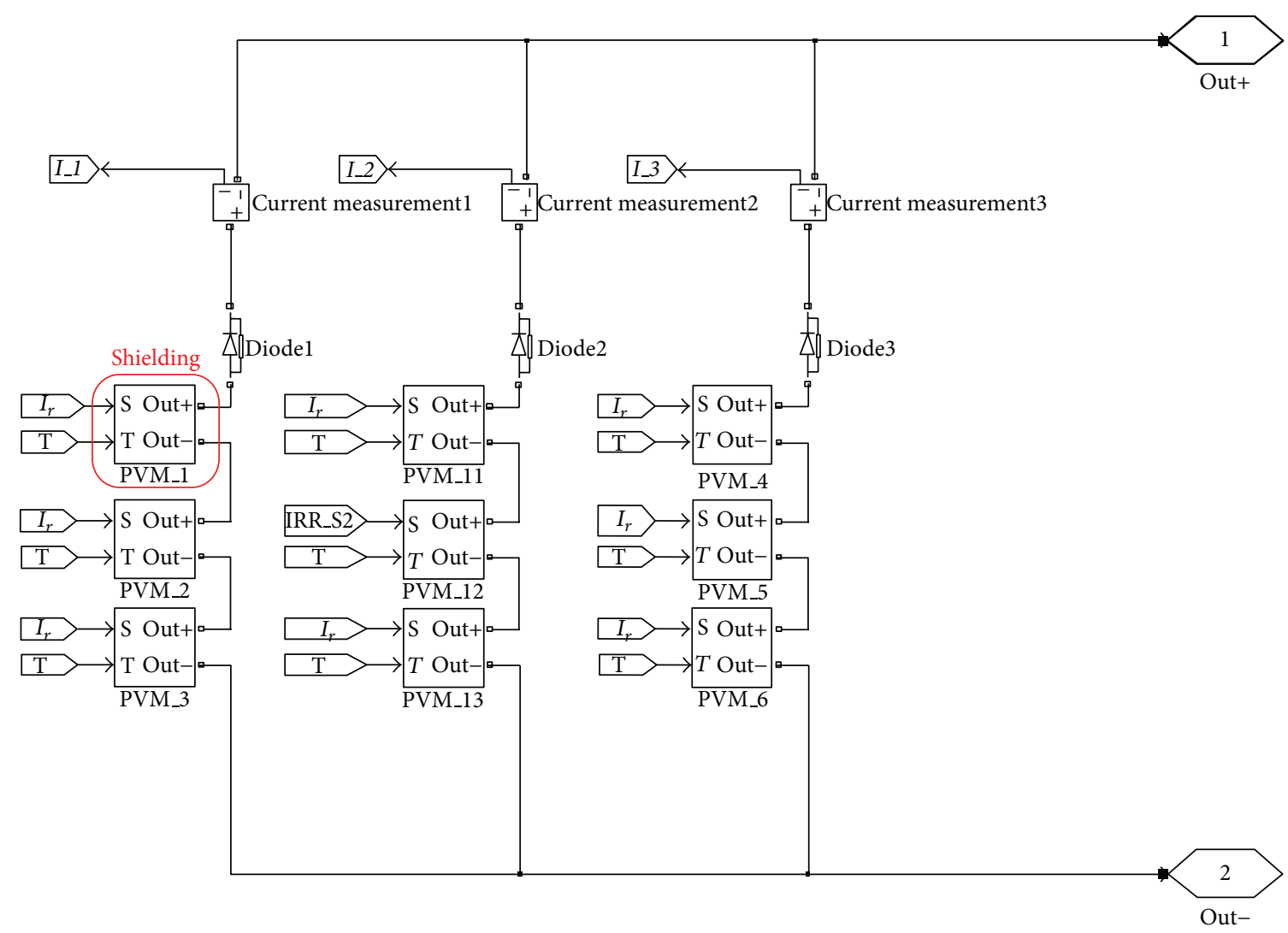

FIGURE 10: One solar module shaded.

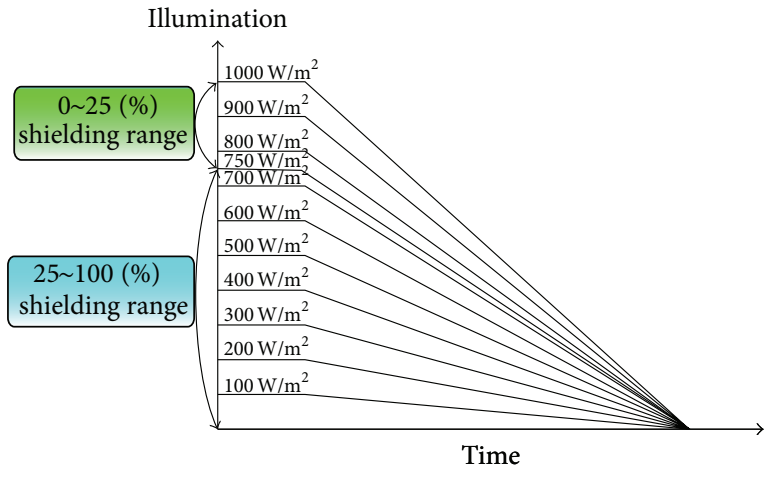

FIGURE 11: Illuminance input training shade method.

4.3. Analysis of Experimental Data of Failure Diagnosis. We are simulate eight panels of the solar array is $1000 \mathrm{~W} / \mathrm{m}^{2}$ illuminance and the illuminance declines at a fixed slope, and the otherwise the illuminance of one solar panel is set as $100 \%$, $95 \%, 85 \%, 75 \%, 70 \%, 65 \%, 55 \%, 45 \%, 35 \%$, and $25 \%$ of the illuminance of the aforesaid eight modules, are imported into this solar panel in turn. Afterwards, the electric energy data of nine solar panels in different illumination combinations are removed and imported into the shading failure distinguishing program according to the data format of SVM of this system for data identification and result output.
At present, the second string of solar modules is used as experimental subject to validate the training result of SVM. This system defines $100 \% \sim 75 \%$ illumination as the normal state and $75 \% \sim 0 \%$ illumination as the abnormal state. The experimental results are as shown in Table 3.

\section{Conclusion}

This paper has completed a monitoring system based on a cloud structure. In terms of a data acquisition system, the data acquisition program of this system has been successfully applied, the smart meter is connected via USB-to-RS-485, packet transfer is instructed, the electric meter responded power consumption data packet is received via Modbus communication protocol, the feedback content is analyzed, and the packet is split. The data are uploaded to the data center according to the database field format and order of this system and displayed at the local end.

In terms of a streaming server, a program with a video receiving function and streaming function has been successfully developed. The streaming output encoding mode, IP address, and Port number can be set in the program. If the client-side wants to watch real-time video, the IP address and Port number can be given to the client-side by a server-side inquiry using a database, and then the client-side can watch real-time video upon connection.

In terms of a cloud system, this system is implemented using IIS with ASP.NET and SQL database technology. It 


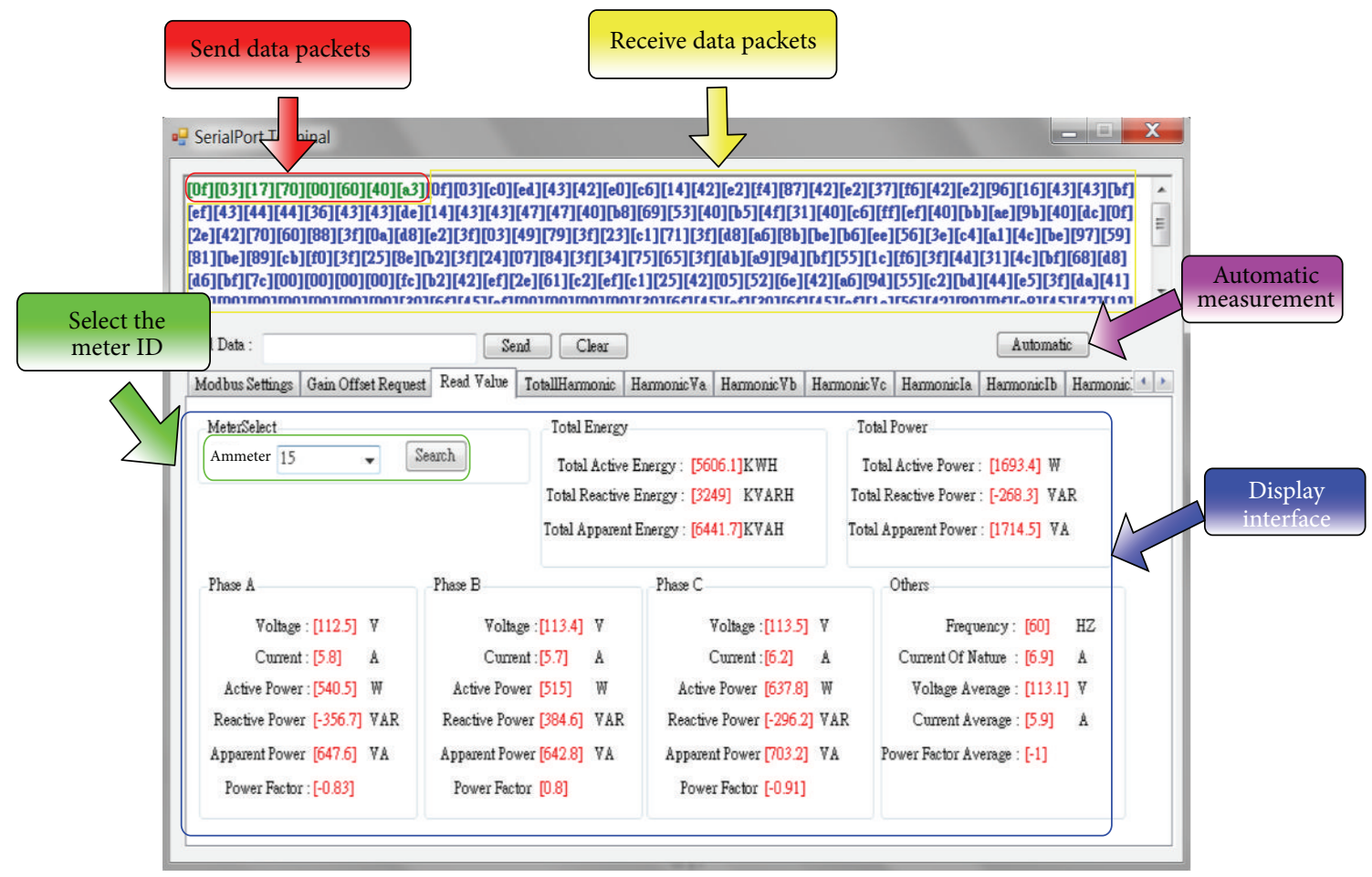

FIGURE 12: Three-phase voltage and current and electric energy information interface.

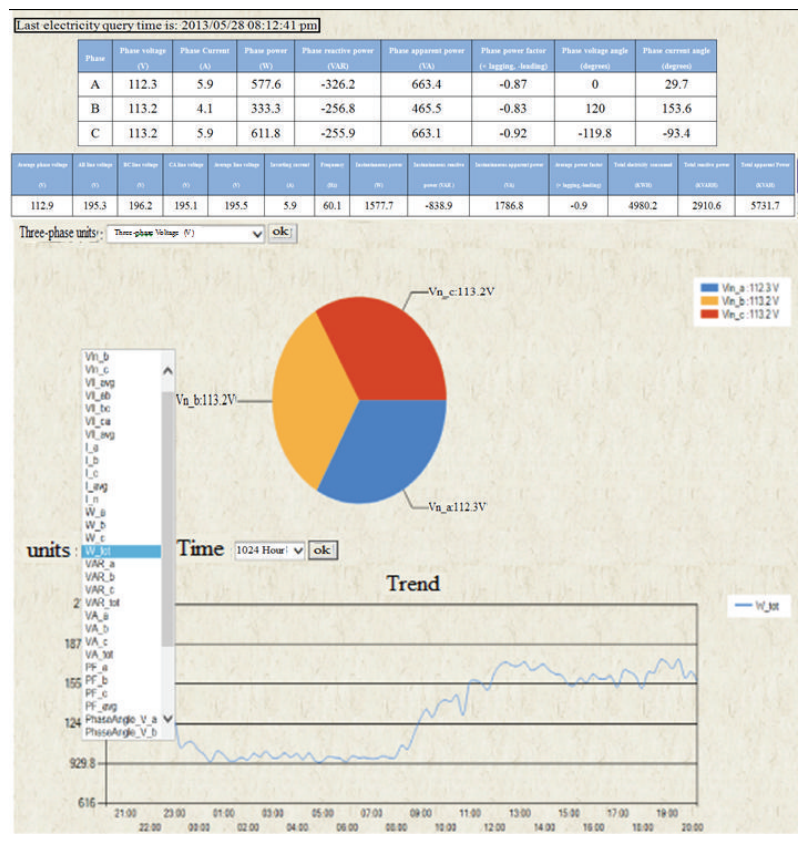

FIGURE 13: Laboratory power consumption inquiry.

has been run in real systems at present, providing window graphical administrator related interface to maintain the overall system integrity, instancy, and fluency, in order that users can use this system, via the network, anywhere at any time. This system is characterized by cross-platform browsing and is more convenient than general monitoring

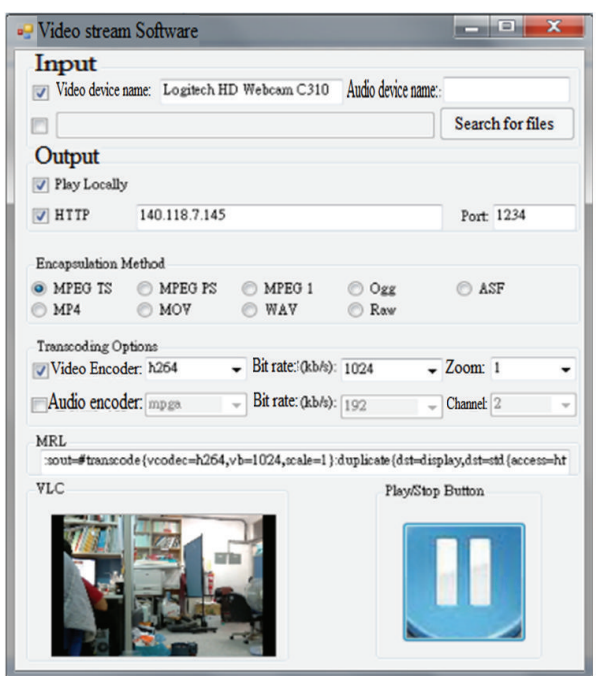

FIGURE 14: Video capture and streaming program.

systems. In addition, it has been integrated with GIS and realtime power consumption information, where the graphical representation prevents the users from searching for their wanted content in multifarious text, thus enabling the user to receive information quickly and clearly. This system not only provides historical information search, but also provides report sending and download functions, thus facilitating the users to view and research measured data. 
TABLE 3: Illumination level identification results.

\begin{tabular}{lcccccccccc}
\hline Illumination level & $100 \%$ & $95 \%$ & $85 \%$ & $75 \%$ & $70 \%$ & $65 \%$ & $55 \%$ & $45 \%$ & $35 \%$ & $25 \%$ \\
Number of data & 322 & 322 & 320 & 320 & 318 & 319 & 317 & 316 & 315 & 318 \\
\hline \multirow{2}{*}{ Normal } & $100 \%$ & $100 \%$ & $99.69 \%$ & $95.31 \%$ & $1.26 \%$ & $0.31 \%$ & $0 \%$ & $0.32 \%$ & $0.63 \%$ & $0.94 \%$ \\
& $(322 / 322)$ & $(322 / 322)$ & $(319 / 320)$ & $(305 / 320)$ & $(4 / 318)$ & $(1 / 319)$ & $(0 / 317)$ & $(1 / 316)$ & $(2 / 315)$ & $(3 / 318)$ \\
First string shaded & $0 \%$ & $0 \%$ & $0 \%$ & $0 \%$ & $0 \%$ & $0 \%$ & $0 \%$ & $0 \%$ & $0 \%$ & $0 \%$ \\
& $(0 / 322)$ & $(0 / 322)$ & $(0 / 320)$ & $(0 / 320)$ & $(0 / 318)$ & $(0 / 319)$ & $(0 / 317)$ & $(0 / 316)$ & $(0 / 315)$ & $(0 / 318)$ \\
Second string shaded & $0 \%$ & $0 \%$ & $0.31 \%$ & $4.69 \%$ & $98.74 \%$ & $99.69 \%$ & $100 \%$ & $99.68 \%$ & $99.36 \%$ & $99.06 \%$ \\
& $(0 / 322)$ & $(0 / 322)$ & $(1 / 320)$ & $(15 / 320)$ & $(314 / 318)$ & $(318 / 319)$ & $(317 / 317)$ & $(315 / 316)$ & $(313 / 315)$ & $(315 / 318)$ \\
\multirow{2}{*}{ Third string shaded } & $0 \%$ & $0 \%$ & $0 \%$ & $0 \%$ & $0 \%$ & $0 \%$ & $0 \%$ & $0 \%$ & $0 \%$ & $0 \%$ \\
& $(0 / 322)$ & $(0 / 322)$ & $(0 / 320)$ & $(0 / 320)$ & $(0 / 318)$ & $(0 / 319)$ & $(0 / 317)$ & $(0 / 316)$ & $(0 / 315)$ & $(0 / 318)$ \\
\hline
\end{tabular}

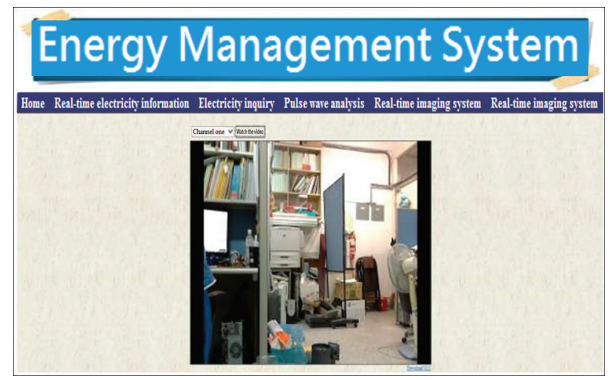

FIgURE 15: Real-time video.

According to the data in Table 3, the illumination shading diagnosis system is designed according to the SVM theory and actually implements early warning.

\section{Conflict of Interests}

The authors declare that there is no conflict of interests regarding the publication of this paper.

\section{Acknowledgment}

The research was supported by the National Science Council of the Republic of China under Grant no. NSC 102-2221-E011-045.

\section{References}

[1] R. D. Tapakis and A. G. Charalambides, "Monitoring cloud motion in cyprus for solar irradiance prediction," Conference Papers in Energy, vol. 2013, Article ID 320618, 6 pages, 2013.

[2] H. Fang, B. Chen, H. Ma, and L. Zhang, "Intelligent monitoring and predicting output power losses of solar arrays based on particle filtering," Mathematical Problems in Engineering, vol. 2013, Article ID 819379, 7 pages, 2013.

[3] T. Watson, G. Reynolds, D. Wragg, G. Williams, and D. Worsley, "Corrosion monitoring of flexible metallic substrates for dyesensitized solar cells," International Journal of Photoenergy, vol. 2013, Article ID 791438, 8 pages, 2013.

[4] C. Ranhotigamage and S. C. Mukhopadhyay, "Field trials and performance monitoring of distributed solar panels using a lowcost wireless sensors network for domestic applications," IEEE Sensors Journal, vol. 11, no. 10, pp. 2583-2590, 2011.
[5] A. Coleman and J. Zalewski, "Intelligent fault detection and diagnostics in solar plants," in Proceedings of the 6th IEEE International Conference on Intelligent Data Acquisition and Advanced Computing Systems: Technology and Applications (IDAACS '11), pp. 948-953, Prague, Czech Republic, September 2011.

[6] A. Drews, A. C. de Keizer, H. G. Beyer et al., "Monitoring and remote failure detection of grid-connected PV systems based on satellite observations," Solar Energy, vol. 81, no. 4, pp. 548-564, 2007.

[7] G. M. Masters, Renewable and Efficient Electric Power Systems, John Wiley \& Sons, New York, NY, USA, 2004.

[8] K. Ding, X. Bian, H. Liu, and T. Peng, "A MATLAB-simulinkbased PV module model and its application under conditions of nonuniform irradiance," IEEE Transactions on Energy Conversion, vol. 27, no. 4, pp. 864-872, 2012.

[9] U. B. Parikh, B. Das, and R. P. Maheshwari, "Combined waveletSVM technique for fault zone detection in a series compensated transmission line," IEEE Transactions on Power Delivery, vol. 23, no. 4, pp. 1789-1794, 2008.

[10] C. Koley, P. Purkait, and S. Chakravorti, "Wavelet-aided SVM tool for impulse fault identification in transformers," IEEE Transactions on Power Delivery, vol. 21, no. 3, pp. 1283-1290, 2006.

[11] J. Ni, C. Zhang, and S. X. Yang, "An adaptive approach based on KPCA and SVM for real-time fault diagnosis of HVCBs," IEEE Transactions on Power Delivery, vol. 26, no. 3, pp. 19601971, 2011.

[12] A. Shintemirov, W. Tang, and Q. H. Wu, "Power transformer fault classification based on dissolved gas analysis by implementing bootstrap and genetic programming," IEEE Transactions on Systems, Man and Cybernetics C: Applications and Reviews, vol. 39, no. 1, pp. 69-79, 2009.

[13] C.-J. Wang, Build power transformer failure diagnosis system by using GSM data acquisition module and multilayer SVM classifier [M.S. thesis], National Kaohsiung University of Applied Sciences, Kaohsiung, Taiwan, 2005. 


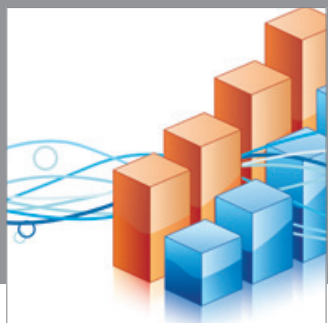

Advances in

Operations Research

mansans

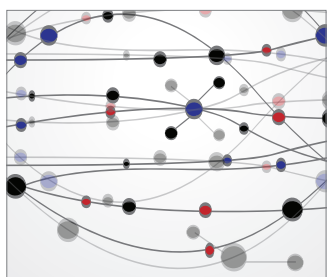

The Scientific World Journal
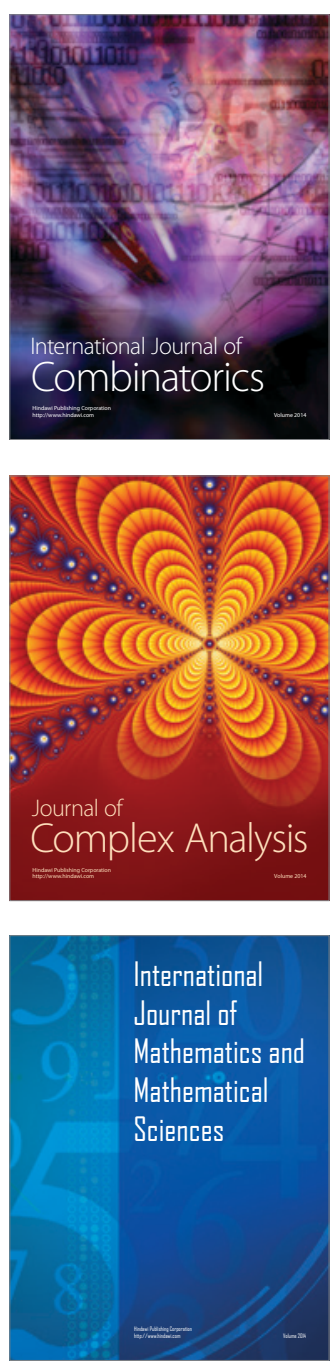
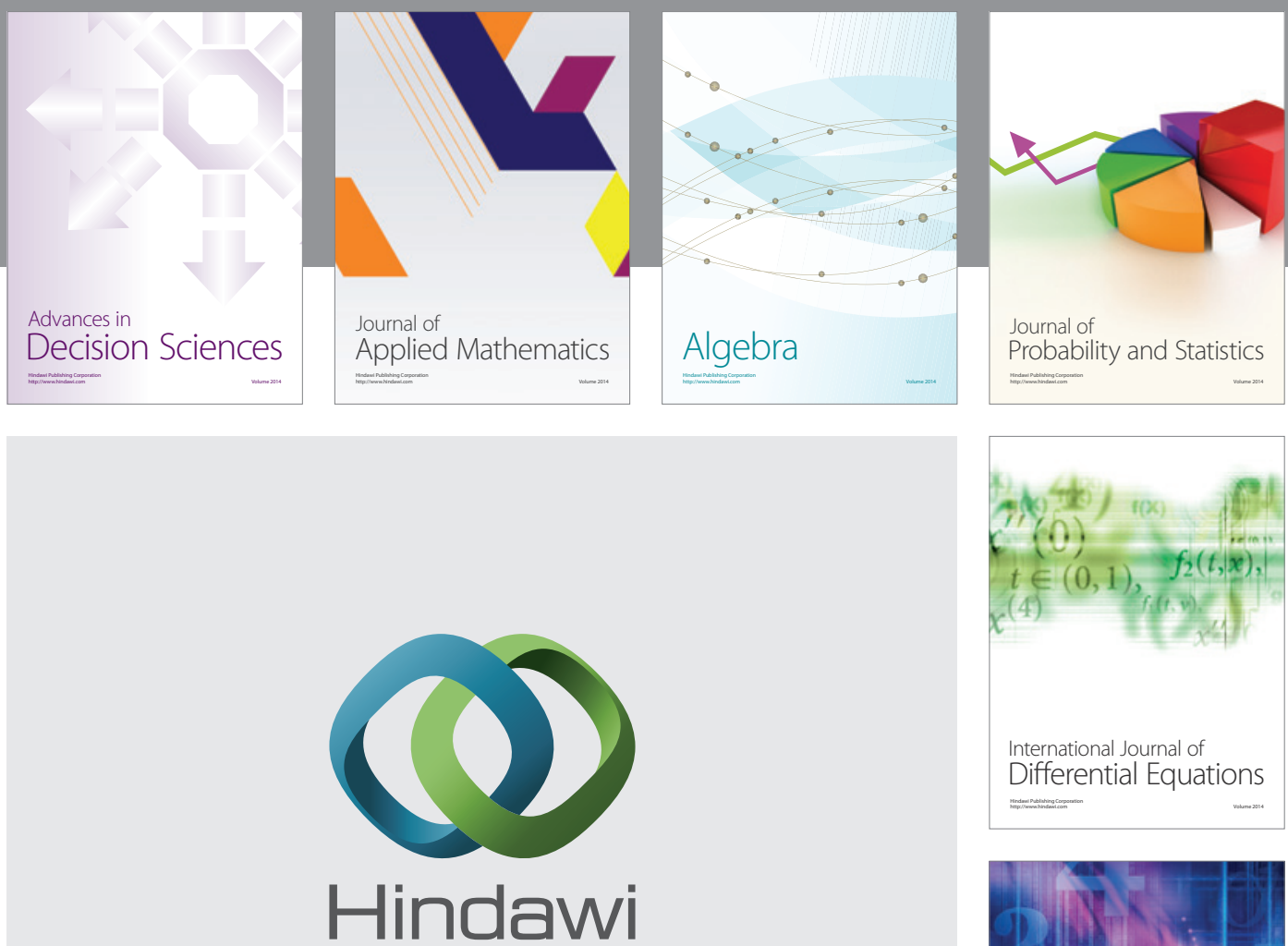

Submit your manuscripts at http://www.hindawi.com
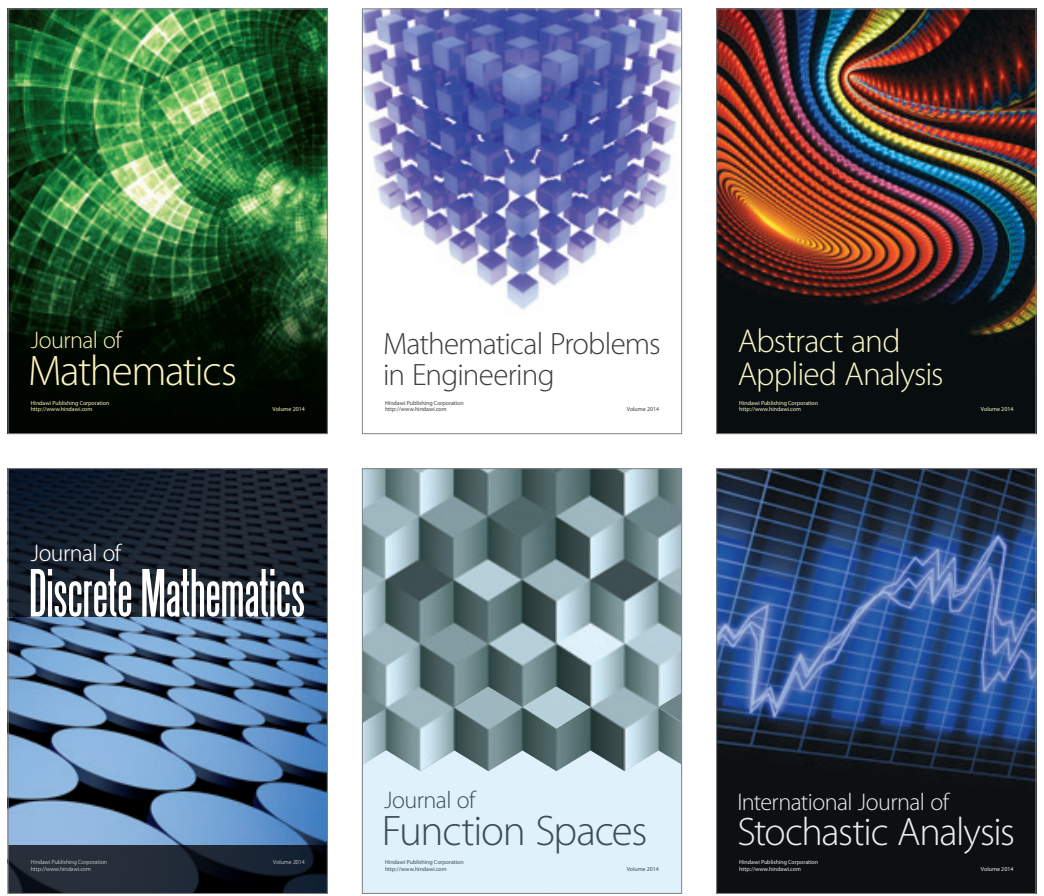

Journal of

Function Spaces

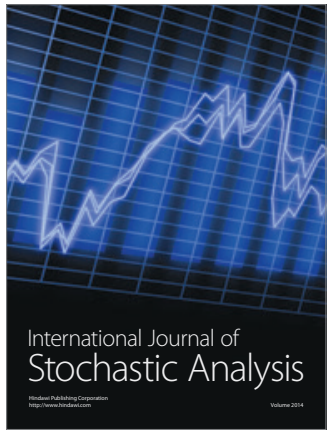

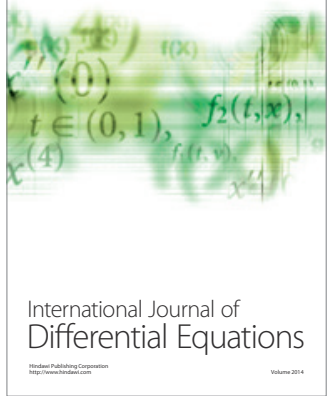
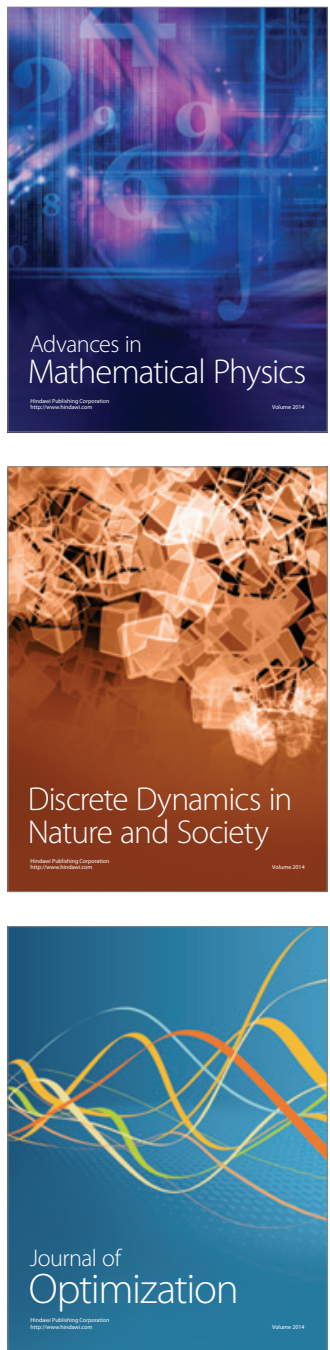